Laser Chem., 1999, Vol. 19, pp. 11-18

Reprints available directly from the publisher Photocopying permitted by license only
(C) 1999 OPA (Overseas Publishers Association) N.V. Published by license under the Harwood Academic Publishers imprint, part of The Gordon and Breach Publishing Group.

\title{
TIME-FREQUENCY RESOLVED COHERENT RAMAN SCATTERING FROM MOLECULAR LIQUIDS AND THEIR MIXTURES WITH QUASI-CW NOISY LIGHT: I $^{(2)}$ CRS SPECTROGRAMS
}

\author{
DARIN J. ULNESS, MICHAEL J. STIMSON, \\ JASON C. KIRKWOOD and A. C. ALBRECHT* \\ Department of Chemistry, Baker Laboratory, Cornell University, \\ Ithaca, NY 14853
}

(Received 7 April 1997)

It is demonstrated how time-frequency resolved coherent Raman scattering (CRS) signals generated by broadband, non-transform limited, quasi-cw (noisy) light can be sensitive probes of molecular vibrational dynamics. The coherent Raman scattering signals from molecular liquids and their mixtures with noisy light are dispersed onto a CCD array and probed interferometrically to produce time-frequency domain spectrograms. These spectrograms offer an extensive oversampling of the data resulting in improved precision of measured parameters over previous noisy light methods. This technique has been very useful in measuring small changes in material parameters, such as Raman frequency shifts and linewidth changes, in dilution series with Raman inactive diluents. Very recently theory and experiment have extended to include mixtures with multiple Raman resonances. Several examples of experiments are presented and discussed.

Keywords: Coherent Raman scattering; time-resolved spectra; molecular liquids

\section{INTRODUCTION}

Coherent Raman scattering (CRS) has long been a useful probe of condensed phase dynamics providing information on Raman frequencies $\left(\omega_{R}\right)$, line widths $\left(\gamma_{R}\right)$ and hyperpolarizabilities. 'Conventional'

*Corresponding author. 
CRS is a frequency domain technique in which the signal intensity is measured as a function of the frequency difference between two narrowband laser sources. The advent of ultrashort light pulses has allowed measurement of CRS signals in the time domain-time resolved CRS (TR-CRS). Ultrashort pulses are not, however, the only tool allowing for short spectroscopic time resolution. Some time ago [1], it was discovered that broadband, non-transform limited, quasi-cw (noisy) light sources could provide interferometric time resolution that is governed by the correlation time of the noisy light, $\tau_{c}$, and not by the temporal profile of the pulse which is typically nanoseconds long (but could in principle be $\mathrm{cw}$ ). Noisy light interferometry typically involves a single broadband source, which is directed into a dispersion compensated Michelson interferometer, where it is split into identical twin broadband beams. One beam, $\mathbf{B}^{\prime}$, is delayed in the interferometer by a time $\tau$ relative to the other beam $\mathrm{B}$. This noisy light has found many applications [2] including CRS [3] (referred to as I ${ }^{(2)} \mathrm{CRS}$ - the I stands for interferometric and the superscript (2) indicates that the CRS signal is generated in part by two perturbative actions of the broadband fields). The (third order) $\mathrm{I}^{(2)} \mathrm{CRS}$ signal is produced by the in-phase actions of $\mathrm{B}$ and $\mathrm{B}^{\prime}$ and the out-of-phase action of a narrowband field $\mathrm{M}$. $\mathrm{M}$ is effectively monochromatic with frequency $\omega_{M}$ chosen to differ from the central broadband frequency $(\bar{\omega})$ by roughly $\omega_{R}$. When $\omega_{M}$ is to the red (blue) of $\bar{\omega}$ one has coherent antiStokes (Stokes) scattering. The three beams (B, $\mathbf{B}^{\prime}$ and $\left.\mathbf{M}\right)$ are focussed into the sample in the BOX geometry [4]. The resultant $\mathrm{I}^{(2)} \mathrm{CRS}$ signal then propagates along its own unique $k$-vector and can be coarsely spatially filtered from the fundamental beams. Frequency resolving (i.e., 'monochromatically' detecting at a frequency $\omega_{D}$ ) this $\mathrm{I}^{(2)} \mathrm{CRS}$ signal leads to striking interferograms which contain damped, so-called "radiation difference oscillations" (RDOs) [5]. These RDOs oscillate with a frequency $\Delta \equiv \omega_{M} \pm 2 \omega_{R}-\omega_{D}$ (" + ' for anti-Stokes scattering and ' - ' for Stokes scattering). The RDOs have several important features: (i) they are damped with rate constant $2 \gamma=2\left(\gamma_{R}+\gamma_{\text {instr. }}\right)$, where $\gamma_{\text {instr. }}$ is the instrument response correction consisting of a nonzero slitwidth of the monochromator and a nonzero bandwidth of the narrowband beam, (ii) the noisy field frequencies are completely absent and (iii) for fixed $\omega_{M}$ and $\omega_{R}, \Delta$ depends only on the position in frequency space at which the signal is detected $\left(\omega_{D}\right)$ and represents a 
strong down-conversion of the Raman mode (when $\omega_{D} \approx \omega_{M}+2 \omega_{R}$ ). This last point is one of the principle advantages of $\mathrm{I}^{(2)} \mathrm{CRS}$ since one may interferometrically extract any given Raman frequency using near "zero" frequency interferometry. This contrasts with the higher interferometric resolution needed in FT-IR and FT-Raman spectroscopy. More explicitly, in $\mathrm{I}^{(2)} \mathrm{CRS}$, the Nyquist sampling criterion is significantly reduced. A dramatic example of this is the down conversion of high energy $\left(\sim 3000 \mathrm{~cm}^{-1}\right) \mathrm{C}-\mathrm{H}$ stretching modes to RDOs from $0-300 \mathrm{~cm}^{-1}$ [6]. Thus, from the $\mathrm{I}^{(2)} \mathrm{CRS}$ interferograms one is able to extract the material parameters $\omega_{R}$ and $\gamma_{R}$. Furthermore it has been shown [7] that when both the Raman resonant and nonresonant contributions to the material response are considered one may also extract the ratio of the nonresonant to resonant hyperpolarizability $(R)$.

Recently [8] $\mathrm{I}^{(2)} \mathrm{CRS}$ has been extended to a two-dimensional timefrequency domain method in which the entire frequency resolved $\mathrm{I}^{(2)} \mathrm{CRS}$ spectrum is detected at every delay line setting throughout a $\tau$ scan. The $\mathrm{I}^{(2)} \mathrm{CRS}$ signal is dispersed onto a CCD array where each pixel of that array acts as a separate narrowband detector. Hence, many hundreds of separate interferograms are simultaneously acquired during a single $\tau$-scan. Such a two-dimensional representation of a coherent Raman signal is referred to as a spectrogram. Although an interferogram for a particular setting of $\omega_{D}$ contains all the material parameters, the very large over-sampling of the signal represented by a spectrogram greatly improves the precision of the extracted parameters. In fact, for certain Raman lines a precision of better than $40 \mathrm{ppm}$. relative standard deviation can be achieved for $\omega_{R}$ measurements.

\section{I $^{(2)}$ CRS SPECTROGRAMS: TWO EXAMPLES}

In Figure 1(a) an $\mathrm{I}^{(2)}$ CARS spectrogram for the 'ring breathing' mode $\left(1002 \mathrm{~cm}^{-1}\right)$ of chlorobenzene is shown. The darker regions represent higher signal intensities. For this spectrogram $\left(\omega_{M} / 2 \pi c\right)$ was set to a fixed value of $15496.51 \mathrm{~cm}^{-1}$. The position where $\Delta=0$ is seen as a dark vertical line near $\omega_{D} / 2 \pi c=17500 \mathrm{~cm}^{-1}$; the exact value of this detected frequency, which bears a fixed relation to $\omega_{R}$ and $\omega_{M}$, is 


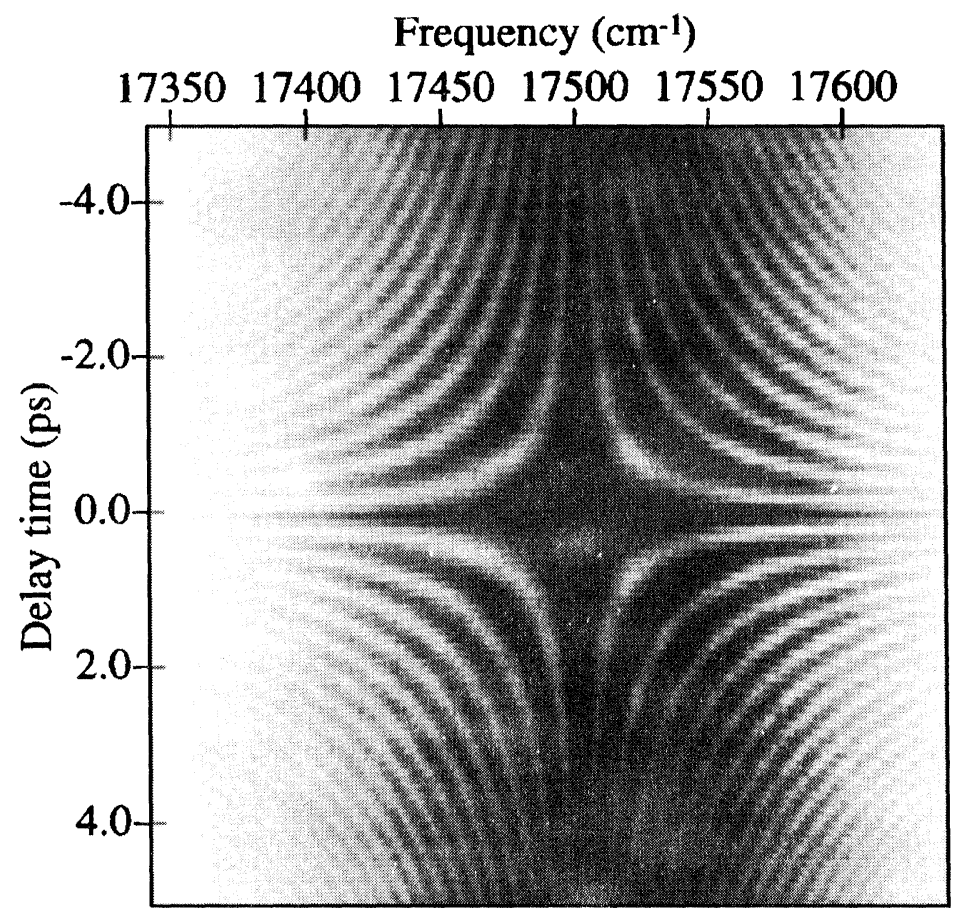

a)

FIGURE 1 (a) The I ${ }^{(2)}$ CARS spectrogram of the $1002 \mathrm{~cm}^{-1}$ 'ring breathing' mode of chlorobenzene. Dark regions indicate higher $\mathrm{I}^{(2)} \mathrm{CARS}$ signal intensities. The narrowband dye laser is tuned to $15496.51 \mathrm{~cm}^{-1}$. The RDOs vanish at $17500.32 \mathrm{~cm}^{-1}$. Parameters obtained from the best fit to this spectrogram are given in the text. (b) The time to frequency Fourier transform of the spectrogram in (a). At least one additional weak Raman mode is seen in the Fourier transform in agreement with the spontaneous Raman spectrum. Only the strong $1002 \mathrm{~cm}^{-1}$ mode was included in the fit.

determined through a full two-dimensional non-linear least squares fit of the complete spectrogram to theory [5, 7]. $\left(\omega_{R} / 2 \pi c\right)$ was found to be $1001.90 \mathrm{~cm}^{-1}$. The parameters $\gamma_{R}=0.127 \mathrm{ps}^{-1}$ and $R=0.062$ are also extracted from the fit. To expose the RDOs contained in the fringes of the spectrogram, the single wavelength interferograms have each been Fourier transformed to frequency space (Fig. 1(b)). Two pronounced diagonal lines are seen. The one labelled ' $\Delta$ ' is the RDO (as $(\Delta / 2 \pi c)$ ) versus $\left(\omega_{D} / 2 \pi c\right)$, and the one labelled ' $-\Delta$ ' is the redundant "negative" frequency component arising from the Fourier transform. These two 


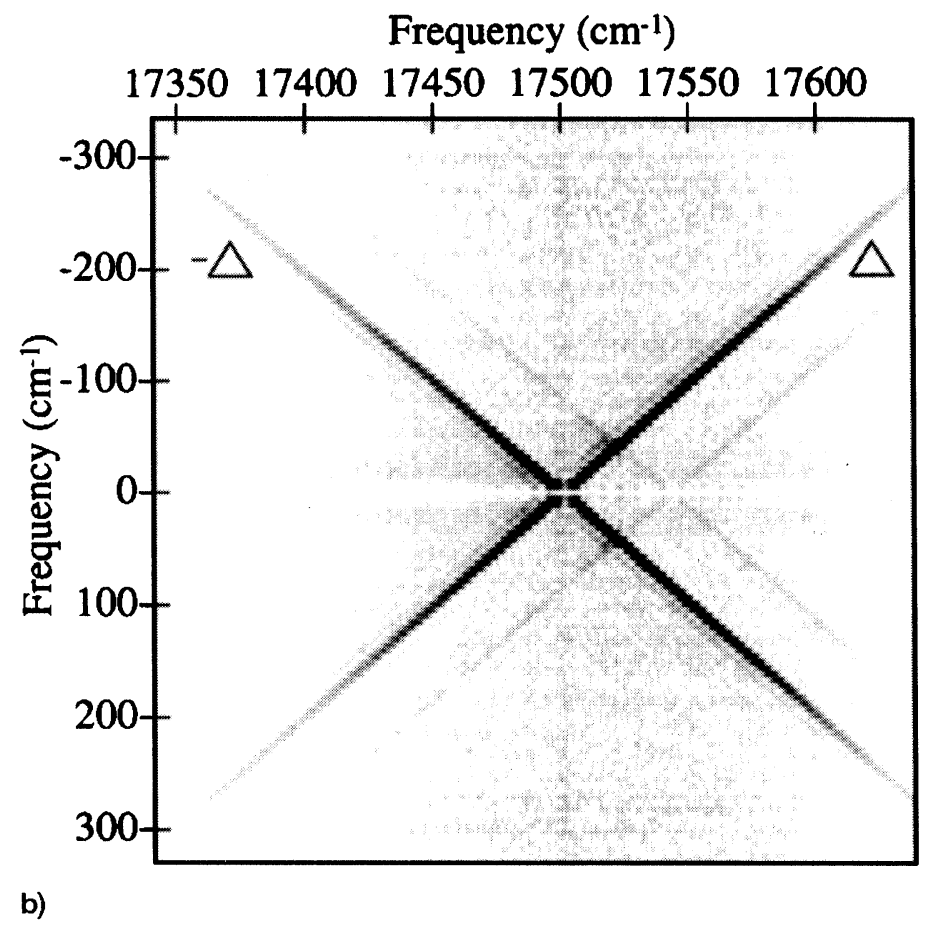

FIGURE 1 (Continued).

diagonal features intersect precisely at the value of $\left(\omega_{D} / 2 \pi c\right)$ for which $\Delta=0$.

At least one additional weak Raman mode is seen in the Fourier transform. The position of the mode(s) agree with the spontaneous Raman spectrum of chlorobenzene. The weak mode(s) was not included in the analysis.

\section{$\mathbf{I}^{(2)}$ CRS of Mixtures}

Dilution series spectroscopy continues to be an important approach to understanding vibrational dynamics in the condensed phase. Often one observes significant changes in the linewidth (the dephasing rate constant) and frequency shifts upon dilution of a Raman active molecular liquid. And these may depend on the nature of the diluent. Time-frequency resolved $\mathrm{I}^{(2)} \mathrm{CRS}$ is proving to be a very useful method 
for such dilution studies. The enhanced precision due to the extensive oversampling allows for precise measurements of small changes in the parameters $\left(\omega_{R}, \gamma_{R}, R\right)$ [9]. The complete theory for the case of a binary mixture consisting of a (single vibration) Raman active molecular liquid and a fully nonresonant diluent liquid has been presented previously [7]. It is seen how the ratio of the nonresonant hyperpolarizability of the diluent to the resonant hyperpolarizability of the Raman active liquid is directly measurable.

Very recently [10], $I^{(2)}$ CRS theory has been extended to include mixtures of an arbitrary number of species each having an arbitrary number of Raman active modes. $I^{(2)}$ CARS spectrograms for the 'ring breathing' modes in mixtures of benzene- $h_{6}\left(992 \mathrm{~cm}^{-1}\right)$ and benzene- $d_{6}$ $\left(944 \mathrm{~cm}^{-1}\right)$ illustrate well a two-mode system. For example, the spectrogram of a $3: 2$ volume ratio $\left(h_{6} / d_{6}\right)$ mixture is shown in Figure 2 (a) - clearly revealing two sets of RDO fringes. There are now two dark vertical features. The one on the right (at $\sim 17525 \mathrm{~cm}^{-1}$ ) is for benzene- $h_{6}$ and the one on the left (at $\sim 17430 \mathrm{~cm}^{-1}$ ) for benzene- $d_{6}$. The strength of the contribution to the $I^{(2)}$ CARS signal of either component in the mixture is governed by the (orientationally averaged) magnitude of its resonant hyperpolarizability and by its mole fraction. Figure 2(b) shows the time Fourier transform of the spectrogram of Figure 2(a), which clearly exposes the two sets of RDOs. The appropriate treatment of such spectrograms is to best fit them to the complete theory [10]. For each mixture one will obtain highly precise measures of the two Raman frequencies, each of their dephasing rate constants and four (not unrelated) nonresonant-toresonant hyperpolarizabilities: $R_{h l l}, R_{h l d}, R_{d / l}$ and $R_{c l d}$. We are currently developing procedures for fitting $I^{(2)}$ CRS spectrograms which contain several Raman resonances.

\section{CONCLUSION}

We have seen that noisy light ean be used to interferometrically probe short time vibrational dynamics through $I^{(2)}$ CRS. Material parameters including the Raman frequency and linewidth and also the ratio of the nonresonant to resonant hyperpolarizability can be extracted from 


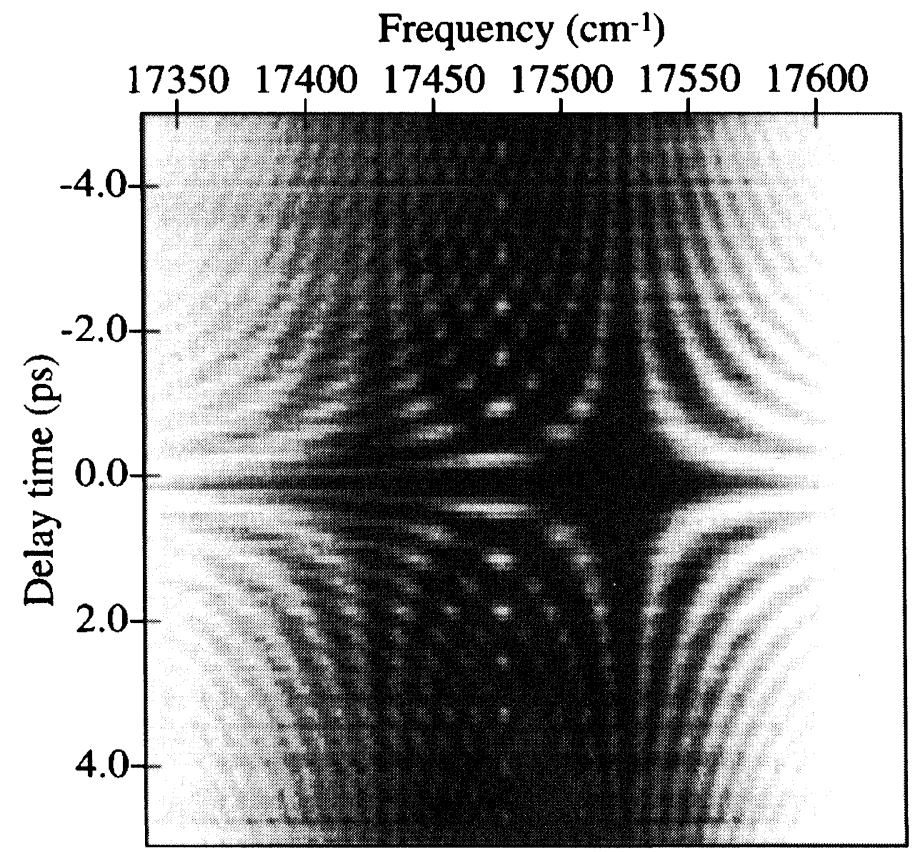

a)

FIGURE 2 (a) The I ${ }^{(2)}$ CARS spectrogram of the 'ring breathing' modes of benzene- $h_{6}$ and benzene- $d_{6}$ for a $3 / 2(\mathrm{v} / \mathrm{v})$ mixture. Dark regions indicate higher $I^{(2)}$ CARS signal intensities. Best fitting theory to such multimode spectrograms is under development. (b) The time to frequency fourier transform of the spectrogram in (a). The two Raman modes are clearly evident.

analysis of the damped radiation difference oscillations seen in the $I^{(2)}$ ('RS interferograms. The precision of these parameters is improved immensely by two-dimensional analysis of I ${ }^{(2)}$ ( RS spectrograms. With this improved precision, small changes in the material parameters that oceur upon dilution can be readily observed by this time-frequency $I^{(2)}$ ( RS technique. It is expected that recent advantees in the theory will allow for an interpretation and subseguent study of more complicated mixture systems. This two-dimensional I (?) $($ RS technique offers an interesting new approatch to recovering datal of considerable importance to licpuid phase dynamics. 


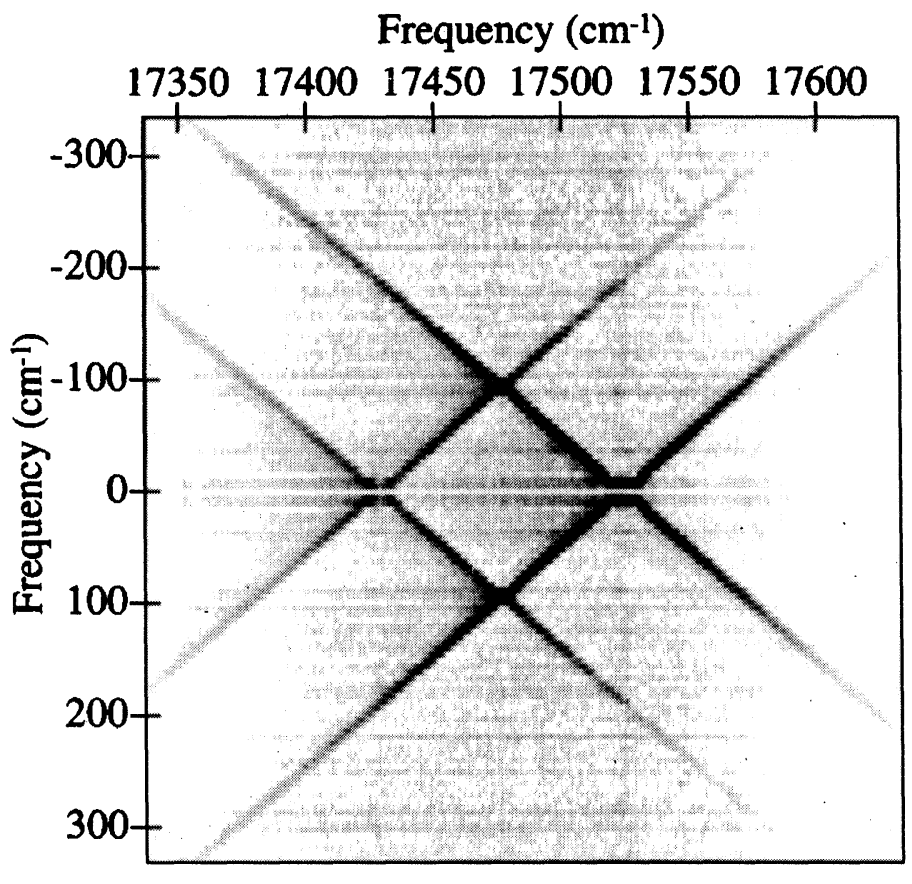

b)

FIGURE 2 (Continued).

\section{References}

[1] Morita, N. and Yajima, T. (1984). Phys. Rev. A, 30, 2525.

[2] Kobayashi, T. (1994). Adv. Chem. Phys., 85, 55.

[3] Hattori, T., Teraski, A. and Kobayashi, T. (1987). Phys. Rev. A, 35, 715.

[4] Shirley, J. A., Hall, R. J. and Eckbreth, A. C. (1980). Opt. Lett., 5, 380.

[5] Dugan, M. A. and Albrecht, A. C. (1991). Phys. Rev. A, 43, 3877.

[6] Ulness, D. J., Stimson, M. J., Kirkwood, J. C. and Albrecht, A. C. (1997). J. Phys. Chem., 101, 4587.

[7] Schaertel, S. A. and Albrecht, A. C. (1994). J. Raman Spectrosc., 25, 545.

[8] Stimson, M. J., Ulness, D. J. and Albrecht, A. C. (1996). Chem. Phys. Lett., 263, 185.

[9] Stimson, M. J., Ulness, D. J. and Albrecht, A. C. (1997). Chem. Phys., 222, 17.

[10] Jason C. Kirkwood, Ulness, D. J., Stimson, M. J. and Albrecht, A. C., Phys. Rev. $A, \mathbf{5 7}, 1417$. 